UDC: $004.738 .5: 81 ' 255$

DOI: https:/ / doi.org/10.18485/beoiber.2020.4.1.6

\author{
Jelena Borljin1 \\ Doctoranda en la Universidad Pablo de Olavide \\ España
}

\title{
LA SUBTITULACIÓN AMATEUR DE SERIES: EL CASO DE FRIENDS
}

\begin{abstract}
Resumen
Gracias a Internet podemos acceder a la llamada subtitulación amateur. La principal ventaja de este fenómeno es, sin duda, la facilidad de acceso -gratuito y libre- a contenido audiovisual en nuestro idioma materno, mientras que una de sus desventajas es el no saber la procedencia de la fuente -la mayoría de estas traducciones son anónimas- y, por tanto, cuestionar la calidad de la traducción. El objetivo de este trabajo es investigar la calidad de las traducciones amateur que se pueden encontrar en la web y comprobar si respetan las reglas de la traducción audiovisual. Para llegar a una conclusión al respecto, el trabajo analiza la calidad de dos traducciones de subtítulos de este tipo del capítulo «The One with All Thanksgivings» de la serie estadounidense Friends, encontradas en la página web podnapisi.com. A través de un análisis comparativo entre las dos traducciones y aplicando las teorías de la traducción audiovisual, se estudia tanto la veracidad como los aspectos formales de las mismas. Como última finalidad del presente estudio, se quiere demostrar que en Internet pueden encontrarse casos opuestos, es decir, traducciones que sí respetan las reglas de traducción como también aquellas que no, y que no existe una estrategia adecuada o predefinida sobre cómo encontrar una traducción audiovisual amateur fiable y de buena calidad.
\end{abstract}

Palabras clave: traducción, subtitulación amateur, en línea, Internet, Friends.

TV SHOW AMATEUR SUBTITLING: THE CASE OF FRIENDS

\section{Summary}

Thanks to the Internet we can access the so-called amateur subtitling. The main advantage of this phenomenon is, without a doubt, the free access to audiovisual content in our mother tongue, while one of its disadvantages is not knowing the source's origin. Most of these translations are anonymous, and therefore, the quality of the translation is questionable. This paper aims to investigate the quality of amateur translation online, as well as to research whether it respects the rules of audiovisual translation. To reach a conclusion in this regard, the work analyzes the quality of two such translations of the episode "The One

1 jborljin@yahoo.com

BEOIBERÍSTICA Vol. IV / Número 1 (2020) | 95-113 
with All Thanksgivings" of the American series Friends, found on the podnapisi.com website. Through a comparative analysis between the two translations and applying the theories of audiovisual translation, both the veracity and the formal aspects are studied. As a final objective of this study we want to show that the opposite cases can be found on the Internet, that is, both translations that do respect the translation rules as well as those that do not, and that there is no adequate or predefined strategy on how to find a reliable amateur audiovisual translation and of good quality.

Keywords: translation, amateur subtitling, online, Internet, Friends.

\section{Introducción}

Debido a que la accesibilidad a Internet se ha convertido hoy día en algo cotidiano e imprescindible, existen numerosas fuentes de información a nuestro alcance. Sin embargo, la otra cara de la moneda es la fiabilidad de las mismas; con tantos datos a nuestro alrededor resulta difícil distinguir qué fuentes son fiables.

Dentro de este océano de información hay un mar de traducciones audiovisuales. Para Bogucki (2009: 49), Internet es el precursor de un nuevo tipo de traducción audiovisual, la denominada comúnmente como la subtitulación amateur. Precisamente la etimología de la palabra amateur, latín amateur (el que ama) (Littré), destaca el contexto en el que se va a utilizar esta palabra en el presente trabajo.

Internet nos facilita mucho poder disfrutar de las obras del séptimo arte. Podemos encontrar una inmensa cantidad de material audiovisual sin necesidad de pensar en si se ha producido en nuestra lengua o no, ya que están a nuestro alcance a través de páginas piratas, infinidad de traducciones y subtítulos específicos para películas o, incluso, para el ansiado capítulo de nuestra serie que hemos estado esperando durante tanto tiempo. Sin embargo, rara vez nos preguntamos sobre la calidad de estas traducciones, si están bien hechas y si son correctas. Precisamente este es el objetivo que perseguimos con este trabajo: investigar la calidad de las traducciones amateur que se pueden encontrar en la web y averiguar si respetan las reglas de la traducción audiovisual.

Este trabajo va a analizar la calidad de dos traducciones de subtítulos del capítulo 8 de la temporada 5, The One with All Thanksgivings, de la serie estadounidense Friends, encontradas en la página web podnapisi.com. La serie se hizo muy popular desde el primer capítulo. Según Carter (2014) entre 20 y 29 millones de espectadores vieron cada una de las temporadas. Las dos traducciones son elegidas por ser las más descargadas de todas las traducciones ofrecidas en dicha página web (podnapisi.net).

El trabajo aporta un breve resumen teórico en cuanto a la definición de la subtitulación, las características técnicas (la extensión máxima, la regla de los seis segundos, las reglas ortográficas) y la subtitulación amateur y continua con el análisis comparativo de las dos traducciones. 


\section{La subtitulación}

La subtitulación es una de las modalidades centrales de la traducción audiovisual y en la que, dada su complejidad, es necesario tener en cuenta muchos componentes que juegan un papel muy importante a la hora de transmitir el mensaje adecuadamente. Díaz Cintas (2003: 32) define la subtitulación como «[...] una práctica lingüística que consiste en ofrecer, generalmente en la parte inferior de la pantalla, un texto escrito que pretende dar cuenta de los diálogos de los actores [...]». El mismo autor enfatiza que a un material audiovisual subtitulado lo determinan tres características o componentes fundamentales que deben estar perfectamente coordinados: la palabra oral, la imagen y los subtítulos. Chaume (2004: 33), por su parte, explica este tipo de traducción audiovisual como «[...] un texto escrito [...] en la lengua meta a la pantalla en donde se exhibe una película en versión original, de modo que estos subtítulos coincidan aproximadamente con las intervenciones de los actores de la pantalla», mientras que Baker (1998: 244-245) lo define de una manera más sencilla concluyendo que la subtitulación es la transcripción de una película o un diálogo de televisión presentada simultáneamente en la pantalla.

\subsection{Características técnicas}

Podemos encontrar varios estudios sobre subtitulación en los que diferentes autores escriben sobre los aspectos técnicos de este tipo de traducción, pero todos ellos coinciden en la extensión de cada subtítulo: un máximo de dos líneas de 40 caracteres cada una donde la línea superior debe ser de una longitud menor que la inferior por la contaminación de la imagen (Castro Roig 2001: 280). Es más, Díaz Cintas (2001: 27) afirma que «en cuanto al formato vídeo y DVD, el número es de unos 32 a 35 caracteres». Sin embargo, nuestro trabajo se ha limitado a 40 al tratarse de traductores amateurs.

Muchos autores (Agost 1999: 18, Díaz Cintas 2001: 27-28, Castro Roig 2001: 278) también subrayan la necesidad y obligación de respetar la regla de los seis segundos, según la cual en este período de tiempo el espectador (medio) es capaz de leer y asimilar la información contenida en dos líneas de subtítulos. Lo que Geogakopoulou (2009: 22) afirma es que no importa si la traducción es perfecta o no en el sentido de su contenido, pues siempre va a ser un fracaso si el espectador no tiene el tiempo suficiente para leerla.

La traducción audiovisual para la subtitulación debe respetar unas reglas determinadas respecto a las restricciones de su formato y ubicación en la pantalla. El traductor debe seguir dichas reglas para facilitar a los espectadores la tarea del visionado, permitiéndoles así disfrutar mejor del contenido audiovisual. El público tiene que concentrarse en dos tipos de informaciones: la acción que se desarrolla en la pantalla y la 
traducción de los diálogos, es decir, los subtítulos (Georgakopoulou 2009: 23). Diaz Cintas (2003: 62) sugiere que la sincronía temporal entre los subtítulos y el texto oral es imprescindible.

También, es muy importante respetar la ortografía; los signos de interrogación y de exclamación, las mayúsculas, las tildes, etc. se utilizan del mismo modo que en cualquier otro tipo de texto. Las cursivas se utilizan para destacar las voces en off que no se pueden ver en la pantalla (voces de televisión, radio, la voz del narrador, transcripción de la información que aparece en la imagen, etc.) y los guiones se usan para separar las intervenciones de los diálogos. Respecto a los tres puntos suspensivos iniciales, Díaz Cintas opina que cuando «se encuentran al final del subtítulo indican la continuación del mismo en la proyección siguiente, que comienza bien con tres puntos suspensivos y [que] tienen otra función que consiste en indicar una pausa, omisión o interrupción en el discurso oral del personaje» $(2001: 116,117)$.

\subsection{La subtitulación amateur}

Con las nuevas tecnologías y las posibilidades que Internet nos da, a finales del siglo XX, un nuevo tipo de subtitulación empieza a extenderse: la subtitulación amateur. Este tipo de traducción es realizada por personas, aparentemente, sin la formación adecuada; individuos que traducen el material audiovisual por placer y de manera gratuita. Su objeto es compartir el material con el resto de fans con los que comparten el mismo interés y la misma lengua materna, en la mayoría de los casos (Simó 2005: 32).

\section{Descripción del corpus}

Como ya hemos mencionado, el corpus de análisis son dos traducciones de subtítulos del capítulo 8 de la temporada 5 -The One with All the Thanksgivings- de la serie Friends. La serie se emitió por primera vez el 22 de septiembre de 1994, y hasta el último capítulo, estrenado el 6 de mayo de 20042, fue muy popular (Carter 2014).

Los protagonistas son seis amigos (Rachel, Mónica, Phoebe, Joey, Chandler y Ross) y las distintas vivencias y relaciones de cada uno de ellos en Manhattan, Nueva York. Según Cuéllar y García-Falces (2004: 6), Friends es una comedia disparatada con una gran cantidad de situaciones absurdas, referencias culturales, connotaciones sexuales, dobles sentidos y mucho chiste visual. Es un tipo de humor muy asequible que no requiere ningún esfuerzo por parte del público, aunque no esté familiarizado con la cultura de origen.

2 En IMDB.com 719443 usuarios la valoran con un 8,9 (Friends).

BEOIBERÍSTICA Vol. IV / Número 1 (2020) | 95-113 
Las traducciones al español que conforman este análisis las hemos encontrado en la página web podnapisi.com. El autor de una es anónimo mientras que la otra traducción, al final, contiene el seudónimo Jerus. Para facilitar el estudio y la compresión de este trabajo, distinguiremos entre Traducción1 (de autor desconocido) y Traducción2 (de Jerus). Ambas están adaptadas para el público de América Latina, pues se observan ciertos rasgos del español latinoamericano como la ausencia de uso del pretérito perfecto o del pronombre de la segunda persona del plural vosotros (Matte Bon 2009: 115, 244).

\section{Metodología}

Para abordar los propósitos marcados previamente y analizar correctamente cada una de las traducciones, hemos determinado la comparación como línea de trabajo que mejor ayuda a nuestros objetivos. En lo sucesivo, compararemos las dos traducciones y aplicaremos las teorías de la traducción audiovisual para, finalmente, llegar a formular una conclusión sobre la calidad de dichas traducciones.

\section{Análisis}

\subsection{Análisis de la veracidad de las traducciones}

Saber traducir no solo supone conocer las lenguas origen y meta, sino que también implica ser conocedor de la cultura -ajena y propia-, especialmente si se trata de la traducción del humor. Zabalbescoa opina que no solo hay que prestar atención al humor y la transmisión que se haga de este en otra lengua, sino que también hay que mostrar el virtuosismo lingüístico del autor del guion (2001: 260), que precisamente es una de las razones del éxito mundial de la serie Friends.

\subsubsection{Traducción inexacta que provoca la transmisión de un mensaje equivocado}

Chaume Varela (2005: 11) subraya que uno «de los estándares de calidad de una subtitulación es que el texto meta sea fiel al texto origen». En el siguiente ejemplo se puede ver que los traductores del capítulo han interpretado las palabras de Mónica de forma diferente:

Yeah, I mean, I look great.
Mónica:
my heart's not in trouble anymore.




\begin{tabular}{|c|c|c|c|}
\hline 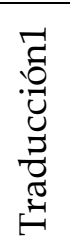 & $\begin{array}{l}\text { 00:14:59,273 --> 00:15:01,367 } \\
\text { Es decir, luzco genial. } \\
\text { 00:15:01,567 --> 00:15:05,288 } \\
\text { Me siento genial, } \\
\text { no tengo problemas amorosos. }\end{array}$ & 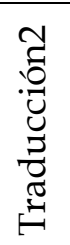 & $\begin{array}{l}\text { 00:14:59,033 --> 00:15:04,613 } \\
\text { Si, si fue grandioso. Si, me sentí genial } \\
\text { y mi corazón no tiene más problemas!. }\end{array}$ \\
\hline
\end{tabular}

Podemos observar que el autor de la primera traducción ha elegido una solución que, desde el punto de vista de un espectador hispanohablante, sí tiene una justificación, ya que puede conectar con la trama del episodio: Mónica ya no está gorda -convirtiéndose esto en la razón por la que ya no tiene más problemas amorosos- y no resulta contradictoria la risa en off que se oye después de su réplica. Sin embargo, eso no es lo que se expresa en la versión original: ella dice que su corazón ya no está en peligro refiriéndose a problemas cardíacos y no a sus relaciones sentimentales. De esta manera, se tergiversa el sentido del mensaje original, especialmente teniendo en cuenta que en la siguiente escena Mónica le pregunta a Rachel cómo debe comportarse si quiere seducir a Chandler y, luego, en dicha seducción, se nota su falta de experiencia en la comunicación con los hombres -resultaría contradictoria su declaración anterior en la que aseguraba que ya no tenía problemas amorosos-.

Considerando lo anterior, la propuesta de la Traducción2 es más afín al texto origen y, por tanto, más lograda, aunque se trata de una traducción literal y contiene la repetición innecesaria de la partícula afirmativa «sí».

\subsubsection{Traducciones sin sentido}

\begin{tabular}{|c|c|c|c|}
\hline & $\begin{array}{ll}\text { Ross: } & \text { You know, maybe } \\
\text { ndler: } & -E n \\
\text { Ross: } & \end{array}$ & \multicolumn{2}{|c|}{$\begin{array}{c}\text { - Emotional Knapsack? Right on. } \\
\text { - Right on. }\end{array}$} \\
\hline 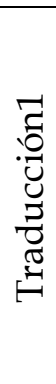 & $\begin{array}{l}\text { 00:11:18,219 --> 00:11:20,313 } \\
\text { Invitaré a salir a Rachel... } \\
\text { 00:11:20,555 --> 00:11:23,183 } \\
<\text { i>...y le cantaré la última } \\
\text { canción.</i> } \\
00: 11: 23,433 \text {--> 00:11:25,731 } \\
\text { ¿"Mochila emocional"? } \\
\text { ¡Seguro! }\end{array}$ & 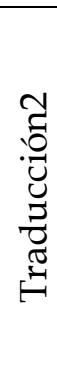 & $\begin{array}{l}00: 11: 18,689 \text {--> 00:11:23,449 } \\
\text { Estoy pensando en invitar a salir a } \\
\text { Rachel esta noche. Y tocarle esa canción } \\
\text { que escribimos la semana pasada. } \\
00: 11: 23,562 \text {--> 00:11:24,688 } \\
\text { "Mochila emocionada?". } \\
\text { 00:11:24,689 --> 00:11:25,085 } \\
\text { Si. }\end{array}$ \\
\hline
\end{tabular}

En este contexto, en el que Chandler quiere dar apoyo a Ross porque éste pretende enseñarle a Rachel su canción, el uso de la palabra seguro no tiene mucho sentido tal y como se nos muestra en el ejemplo de Traducción1. El autor de Traducción2, por su parte, opta por ignorar la reacción de Chandler y no traducir «Right on!». Además, incluye la 
reacción positiva de Ross al incorporar su afirmación. Consideramos, de todos modos, que la traducción más adecuada para la reacción de Chandler sería «iQué guay!» o «iMuy bien!» o cualquier otra expresión con un significado similar.

Los siguientes dos ejemplos nos muestran que cuando el traductor no entiende bien la lengua de origen o no presta demasiada atención al texto original, puede acabar realizando una traducción completamente carente de sentido.

Ejemplo 1:

\begin{tabular}{|c|c|c|c|}
\hline \multicolumn{4}{|c|}{ Monica: } \\
\hline 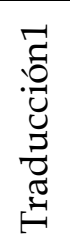 & $\begin{array}{l}\text { 00:07:27,405 --> 00:07:30,284 } \\
\text { No querrás que yo cuente } \\
\text { esta historia. }\end{array}$ & 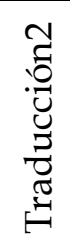 & $\begin{array}{l}\text { 00:07:28,060 --> 00:07:30,620 } \\
\text { Miren, quien de todos ustedes, } \\
\text { no quieren que cuente esta } \\
\text { historia!. }\end{array}$ \\
\hline
\end{tabular}

La propuesta de la Traducción2 llega a confundir a los espectadores porque resulta incomprensible. Sin embargo, la solución de la otra traducción es mucho más aceptable, aunque la primera línea sea más larga, un aspecto que se debe evitar por la contaminación de la imagen, y no esté dividida correctamente. Una posible propuesta sería:

Tú no querrás

que cuente esta historia.

Al incluir el pronombre tú al inicio del subtítulo, se enfatiza el mensaje y resulta una buena opción traslativa para la locución inglesa «Look, of all people, you...». Eliminamos el pronombre yo porque en este ejemplo este tipo de «concisión no perjudica la sintaxis ni el estilo del original» (Díaz Cintas 2001: 126).

Ejemplo 2:

Rachel:

Monica:
I am never going out with him again.

I don't care how much he begs.

I think his begging days are over, now that he's with Nancy Branson. 


\begin{tabular}{|c|c|c|c|}
\hline 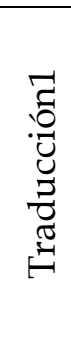 & $\begin{array}{l}\text { 00:09:49,380 --> 00:09:53,635 } \\
\text { No volveré a salir con él. } \\
\text { No me importa cuánto ruegue. } \\
\text { 00:09:53,843 --> 00:09:56,892 } \\
\text { Si está con Nancy, } \\
\text { ya no tiene que rogar. }\end{array}$ & 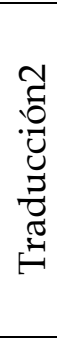 & $\begin{array}{l}\text { 00:09:44,352 --> 00:09:51,517 } \\
\text { Nunca voy a volver a salir con } \\
\text { el de nuevo. No me importa cuanto lo } \\
\text { quiero } \\
\text { 00:09:54,575 --> 00:09:57,522 } \\
\text { Creo que sus días de suplica terminaron } \\
\text { ahora que sale con Nancy Branson }\end{array}$ \\
\hline
\end{tabular}

Los espectadores hispanohablantes no pueden relacionar el comentario de Mónica de la Traducción2 con la parte de súplica que está dirigida al personaje de Rachel porque está mal traducido. «No importa cuánto lo quiero» no equivale a «I don't care how much he begs» y simplemente se pierde el sentido. La solución de la otra traducción es mucho mejor y equivale al original.

En el siguiente ejemplo se ve que el traductor ha transmitido un mensaje que, semánticamente, no tiene sentido:

\begin{tabular}{|c|c|c|c|}
\hline \multicolumn{2}{|r|}{ Ross: } & \multicolumn{2}{|c|}{ So, uh, where's Monica? } \\
\hline 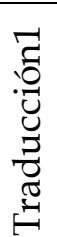 & $\begin{array}{l}\text { 00:13:46,284 --> 00:13:47,285 } \\
\text { ¿Y Monica? }\end{array}$ & 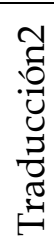 & $\begin{array}{l}\text { 00:13:45,961 --> 00:13:47,398 } \\
\text { Adónde está Monica?. }\end{array}$ \\
\hline
\end{tabular}

Simplemente, esta traducción no es adecuada para esa pregunta. Consideramos que «¿Y Mónica?» es una opción más adecuada para transmitir el mensaje.

García Luque (2005: 68) confirma lo mencionado en el apartado 2. subrayando que «desde el punto de vista de su relación con la imagen, el diálogo cinematográfico se adapta por la siguiente norma: no entrar nunca en contradicción con la imagen [...]». Los siguientes casos demuestran, precisamente, lo contrario, obviando esta norma:

Ejemplo 1:

\begin{tabular}{|r|l|}
\hline Monica: & $\begin{array}{l}\text { I love the way this box feels } \\
\text { against my cheek. }\end{array}$ \\
\hline \multirow{2}{*}{ Traducción2 } & $\begin{array}{l}\text { 00:16:55,691 --> 00:17:03,276 } \\
\text { Me encanta la forma en que esta } \\
\text { caja se siente en mi cuello. }\end{array}$ \\
\hline
\end{tabular}

Se confunde al espectador hispanohablante porque lee la palabra cuello y ve que Mónica está poniendo la caja en su mejilla. 
Ejemplo 2:

\begin{tabular}{|r|l|}
\hline Ross: & $\begin{array}{l}\text { Could you please not do that feet } \\
\text { first? }\end{array}$ \\
\hline Traducción2 2 & $\begin{array}{l}\text { 00:18:15,550 --> 00:18:22,496 } \\
\text { Puede por favor pasar el pie } \\
\text { primero? }\end{array}$ \\
\hline
\end{tabular}

Esta traducción es completamente contraria al original y no corresponde ni al video ni a la continuación de la conversación.

Ejemplo 3:

\begin{tabular}{|r|l|}
\hline Ross: & $\begin{array}{l}\text { You're right, yours is worse. } \\
\text { You are the king of bad } \\
\text { Thanksgivings. }\end{array}$ \\
\hline Traducción1 1 & $\begin{array}{l}\text { 00:03:20,492 --> 00:03:24,417 } \\
\text { Cada vez que lo cuentas, } \\
\text { el tipo tiene más acento. }\end{array}$ \\
\hline
\end{tabular}

Esta versión no solo es incorrecta, sino que tampoco concuerda con los gestos corporales de Ross; las manos levantadas en el aire a la altura del pecho con las palmas extendidas se utilizan normalmente en muchas situaciones para indicar que se dice algo honestamente, es decir, confesar que alguien tiene razón (Knapp 1982: 195).

\subsubsection{Pérdida del humor por la mala traducción}

Como hemos dicho, el humor es el principal factor de esta serie y un arma de doble filo en la misma, pues como ya hemos comentado, no es fácil traducir lo absurdo y lo disparatado. Sin embargo, el factor humorístico debe ser la prioridad del traductor y que éste sea el mismo que se trasmite en la película original (Cuéllar y García-Falces 2004: 6). Díaz Cintas (2001: 132) opina que, si el mensaje no es cómico, entrará en conflicto con la pista sonora original y producirá una confusión en el espectador.

\begin{tabular}{|cc|}
\hline Monica: & I'm gonna spread the legs \\
& as wide as I can. \\
& [JOEY LAUGHING] \\
Monica: & Joey, now is not the time. \\
Joey: & -Yeah. Sorry. Right, sorry \\
\hline
\end{tabular}




\begin{tabular}{|c|c|c|c|}
\hline 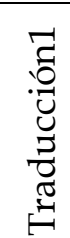 & $\begin{array}{l}\text { 00:06:13,957 --> 00:06:17,006 } \\
\text { Yo abriré las patas tanto } \\
\text { como pueda. } \\
\text { 00:06:20,005 --> 00:06:22,007 } \\
\text { No es el momento. }\end{array}$ & 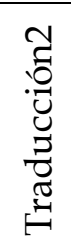 & $\begin{array}{l}\text { 00:06:10,191 --> 00:06:15,329 } \\
\text { Y yo voy a separar } \\
\text { sus piernas lo más que pueda. }\end{array}$ \\
\hline
\end{tabular}

En esta escena, el espectador hispanohablante ni va a entender la comicidad de la conversación entre Mónica y Joey, ni la continuación de la misma («No es el momento»): Joey tiene la cabeza dentro del pavo que el personaje de Mónica está preparando para la cena del Día de Acción de Gracias y no puede sacarla, por lo que Mónica propone la idea que vemos en la versión en inglés. El toque de humor reside en el doble sentido y la connotación sexual3 del texto original, lo que explica la reacción de Joey -su risa- y el consecuente reproche de Mónica -no es el momento para chistes-. La segunda traducción interpreta «legs» como «piernas» pero el humor tampoco se puede reconocer por la presencia del pronombre posesivo «sus» que impide el doble sentido.

El siguiente ejemplo tampoco transmite el humor de la manera adecuada:

\begin{tabular}{|c|c|c|c|}
\hline \multicolumn{4}{|c|}{$\begin{array}{c}\text { Hi, I'm Ross' little sister. } \\
\text { Okay. }\end{array}$} \\
\hline 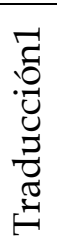 & $\begin{array}{l}\text { 00:08:43,103 --> 00:08:44,866 } \\
\text { Soy la hermanita de Ross. }\end{array}$ & 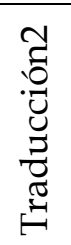 & $\begin{array}{l}\text { 00:08:43,098 --> 00:08:45,071 } \\
\text { Hola, Soy la hermana } \\
\text { menor de Ross. } \\
\text { 00:08:45,845 --> 00:08:47,457 } \\
\text { Okay... }\end{array}$ \\
\hline
\end{tabular}

En este ejemplo el personaje de Mónica (hermana menor de Ross) se presenta a Chandler con la frase «I'm Ross' little sister» lo que provoca el comentario irónico de éste haciendo referencia a su obesidad. La Traducción2 no está clara para los espectadores hispanohablantes porque se oye la risa en off sin saber realmente por qué, pues se pierde la ironía del comentario. La solución de la Traducción1, «hermanita», se acerca un poco más a la broma original, pero no termina de sonar natural en castellano. Consideramos que otras opciones como «hermana pequeña» enfatizarían más la oposición entre palabras e imagen y ayudarían a entender la ironía de Chandler y el humor de su comentario. Además, la expresión «hermana pequeña» suena más natural y equivale perfectamente a la versión en inglés «little sister».

3 Mónica usa la oración I'm gonna spread the legs as wide as I can refiriéndose al pavo, mientras Joey lo entiende en un contexto sexual como si se refiriera a sí misma. 
En el siguiente fragmento podemos ver la omisión del humor por la decisión del traductor de no traducir algunos segmentos:

\begin{tabular}{|c|c|c|c|}
\hline \multicolumn{4}{|c|}{$\begin{array}{c}\text { Hey, I wasn't the only one } \\
\text { who looked like an idiot, all right? } \\
\text { Remember when Ross tried to say } \\
\text { "butternut squash"... } \\
\text {...and it came out, "squatternut bosh"? }\end{array}$} \\
\hline 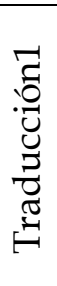 & $\begin{array}{l}\text { 00:06:51,286 --> 00:06:54,130 } \\
\text { ¿Y cuándo Ross quiso decir } \\
\text { "nuez machacada"... } \\
\text { 00:06:54,289 --> 00:06:57,463 } \\
\text {...y dijo } \\
\text { "chuez nachacada"? }\end{array}$ & 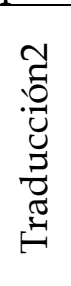 & $\begin{array}{l}\text { 00:06:48,427 --> 00:06:58,105 } \\
\text { No era el unico que parecía un idiota. } \\
\text { Se acuerdan cuando Ross quiso decir } \\
\text { "Butternut squash" y dijo "Squatternut } \\
\text { Buash" }\end{array}$ \\
\hline
\end{tabular}

En la Traducción2 no se llega a comprender el humor de esa réplica por parte del personaje de Joey al no traducirse el chiste, como sí ocurre en la primera.

A continuación, podemos observar una referencia cultural que Chandler usa para provocar de esta forma el humor:

\begin{tabular}{|c|c|c|c|}
\hline \multicolumn{4}{|c|}{ Chandler: } \\
\hline 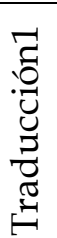 & $\begin{array}{l}\text { 00:19:49,647 --> 00:19:52,776 } \\
\text { Eso no seca las lágrimas } \\
\text { que derramé. }\end{array}$ & 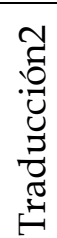 & $\begin{array}{l}00: 19: 49,795-->00: 19: 53,919 \\
\text { Si, bueno, el perdon no trae devuelta } \\
\text { el pequeño niño que lloro todo el } \\
\text { camino a casa! }\end{array}$ \\
\hline
\end{tabular}

En la versión original, Chandler hace referencia a The Little Piggy, una canción para niños sobre los dedos. En la Traducción2 el culturema se pierde y, por tanto, el tono humorístico también. La solución aportada en la versión de la Traducción1 es satisfactoria, pero no óptima. En español existe una canción infantil con la misma temática $\mathrm{y}$, probablemente, sería mejor opción de traducción para provocar el mismo efecto que produce en inglés. Proponemos la traducción Eso no va a devolver al gordito que se comió el huevo como la más natural para los hispanohablantes. 


\subsubsection{Pérdida de la naturalidad en el habla cotidiana en la traducción española}

Díaz Cintas opina que «un trabajo es óptimo cuando el espectador tiene la impresión de que no se le ha escamoteado información alguna» (2001: 126). Los casos anteriores son claros ejemplos de este error. Sin embargo, el espectador también puede tener esta impresión cuando algunas expresiones no suenan natural en su lengua, en este caso en español.

\begin{tabular}{|c|c|c|c|}
\hline \multicolumn{4}{|c|}{$\begin{array}{c}\text { So, uh, Rach... } \\
\text { Does it...? Does it feel weird } \\
\text { around here now? } \\
\text { You know, } \\
\text { since I've been away at college? }\end{array}$} \\
\hline 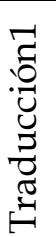 & $\begin{array}{l}\text { 00:09:26,566 --> 00:09:28,614 } \\
\text { ¿Este lugar no está raro... } \\
\text { 00:09:28,860 --> 00:09:32,080 } \\
\text {...desde que me fui } \\
\text { a la universidad? }\end{array}$ & 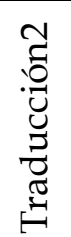 & $\begin{array}{l}\text { 00:09:23,707 --> 00:09:31,551 } \\
\text { Rach? no se, no se siente raro por aca? } \\
\text { Ya sabes, desde que me fui del colegio. }\end{array}$ \\
\hline
\end{tabular}

Aquí la traducción española no provoca el mismo efecto que la versión inglesa. Es decir, no trasmite exactamente el mismo mensaje; Ross elige esta manera de hablar para no ser tan directo, pero lo que le interesa de verdad es saber si Rachel ha notado su ausencia y, además, si le echa de menos. El problema que vemos en la traducción española es que no se percibe el mensaje implícito tras las palabras de Ross, sino que da más bien la impresión de que le interesa si algo ha cambiado en el lugar donde ellos están, donde viven, durante su estancia en otra ciudad.

El uso de calcos4 también influye mucho cuando tenemos la impresión de que se pierde naturalidad en la lengua meta. En las traducciones analizadas se pueden encontrar algunos ejemplos:

\begin{tabular}{|r|l|}
\hline Chandler: & Oh, I'm a duck. I go quack-quack. \\
\hline \multirow{2}{*}{ Traducción1 } & $\begin{array}{l}\text { 00:14:13,895 --> 00:14:15,147 } \\
\text { Soy un pato. Hago "cuac". }\end{array}$ \\
\hline
\end{tabular}

4 Un calco es un préstamo semántico, en que se toma el significado de otro idioma, pero no se crea una palabra nueva, es decir, es la adopción de un significado extranjero para una palabra ya existente en la lengua (Gerding et al. 2018: 177). 
La onomatopeya del pato en español es cua, cua y no cuac, que es precisamente la pronunciación de la versión inglesa quack-quack.

\begin{tabular}{|l|l|}
\hline & 00:00:23,398 --> 00:00:24,940 \\
& $\begin{array}{l}\text { GROUP: } \\
\text { Yeah. Sure. }\end{array}$ \\
\hline Traducción2 2 & $\begin{array}{l}\text { 00:00:23,612 --> 00:00:24,288 } \\
\text { Si, Seguro! }\end{array}$ \\
\hline
\end{tabular}

En este ejemplo observamos un calco obvio. Representa la respuesta de todos a la pregunta «¿Alguien quiere ver la tele?»; al contrario de lo que sucede en inglés, en español no se suele contestar «seguro» como afirmación total a una propuesta. Por ello, proponemos la solución traslativa «Sí, claro»o, incluso, una expresión mucho más coloquial como «iSí, va, va!».

\begin{tabular}{|r|l|}
\hline Pheobe: & Of course, you don't, sweetie. \\
\hline Traducción2 & $\begin{array}{l}\text { 00:04:15,449 --> 00:04:17,888 } \\
\text { Claro que no dulce. }\end{array}$ \\
\hline
\end{tabular}

Al margen de que en la traducción se omita la coma vocativa -por el contexto puede intuirse- la voz inglesa «sweetie» no se emplea en esta ocasión como «dulce», sino como apelativo cariñoso. Una opción mejor sería «cariño», «cielo» o, simplemente, omitirla, como en el otro caso.

\subsubsection{Traducción del título}

La traducción del título del capítulo es imprescindible al ser la primera toma de contacto con el público, lo que influye en más de una ocasión en la decisión de verlo o no. El título de este capítulo en inglés es, según IMDB.com, The One with All the Thanksgivings, o The One with the Thanksgiving Flashbacks, según rottentomatos.com (The One with the Thanksgiving Flashbacks).

Respecto a los títulos de las dos traducciones hay variedad. La Traducción1 nombra al capítulo Todos Los Días De Acciones De Gracias, mientras que la Traducción2 lo hace como The One With All Thanksgivings. Ésta última no solo que no tiene el título traducido, sino que tampoco respeta la ortografía inglesa al tener todas las palabras 
escritas en mayúsculas. La Traducción1 sí lo traduce pero pierde la referencia «the one with...» y que precede a todos los capítulos de la serie tanto en español como en inglés5.

\subsection{Análisis de los aspectos formales de las traducciones}

\subsubsection{Aspectos técnicos}

La Traducción1 respeta las tres reglas mencionadas en el apartado 2.1. (dos líneas, 40 caracteres y seis segundos) pero la Traducción2 no. En primer lugar, se puede ver que en varias ocasiones se escriben tres líneas de subtítulos, contaminando la imagen demasiado:

$$
\text { 00:00:36,897 --> 00:00:41,988 }
$$

Saben que deberíamos hacer? Deberíamos jugar ese juego donde todos dicen una cosa por la cual estan agradecidos.

En segundo lugar, también el número de caracteres es muy superior a 40 , p.ej.:

$$
\text { 00:03:21,398 --> 00:03:25,350 }
$$

Tenes razón. Lo tuyo es lo peor. Eres el rey de los malos días de Accion de Gracia.

Y, en último lugar, no se respeta la regla de los seis segundos:

$$
\text { 00:02:36,668 --> 00:02:39,508 }
$$

No nos iras a contar toda la historia de como tus padres se separaron, no?.

\subsubsection{Normas ortotipográficas}

Como Castro Roig (2001: 279) subraya: «la ortografía debe ser perfecta». Como ya hemos mencionado anteriormente, hay que prestar atención a las tildes, signos de puntuación, mayúsculas, etc. La Traducción2 no respeta mucho las normas ortográficas:

00:00:23,612 --> 00:00:24,288

$\mathrm{Si}$, Seguro!
No hay tilde en Sí ni tampoco hay enunciado exclamativo.

5 En español, siguiendo la guía de episodios de Friendspeich.com, todos los capítulos se nombran de la misma manera con el encabezado «el de» o «el que», según convenga (Guía de episodios). 
00:00:43,936 --> 00:00:47,801

Yo! Yo estoy agradecido por este hermoso descanso que estamos teniendo!.

00:00:59,372 --> 00:01:03,383

$\mathrm{Y}$ Tambien que estoy

agradecido por las tangas.
Falta la puntuación doble «i!».

La palabra Tambien está escrita con mayúsculas sin razón y sin tilde.

En la Traducción1 no hay muchas desviaciones en cuanto a las normas ortográficas, excepto que no se usan símbolos apropiados para los signos de apertura en los enunciados interrogativos o exclamativos:

00:00:21,647 --> 00:00:23,194

żAlguien quiere ver TV?

00:03:35,590 --> 00:03:37,433

`Sí, el mío es peor!

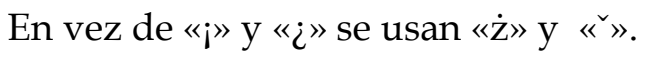

En la parte principal del trabajo hemos citado a Diaz Cinta $(2001: 116,117)$ en cuanto a los puntos suspensivos y se puede concluir que ambas traducciones respetan sus sugerencias.

En cuanto a letra cursiva, solo el traductor de la Traducción1 la usa para indicar «voces en off dentro de secuencia.» Castro Roig (2001: 280):

00:17:30,591 --> 00:17:33,265

Y, si tengo mucho calor...

00:17:34,178 --> 00:17:37,148

$<\mathrm{i}>$...me gusta tomar este cuchillo. $</ \mathrm{i}>$
Mientras el primer subtítulo está en la pantalla, Mónica, que habla en ese momento, no está presente en la misma.

\subsubsection{Normas de estilo}

Un traductor de material audiovisual siempre tiene que pensar en la cohesión del texto en los subtítulos por la restricción de espacio que hemos comentado en capítulos anteriores. Como Castro Roig explica: «lo bueno, si breve, dos veces bueno» (2001: 281). 
Precisamente esa restricción hace difícil traducir los materiales de este tipo. Sin embargo, hay pocas reglas que ayuden a los traductores a reducir el contenido traducido.

Lo que casi siempre se puede omitir son las repeticiones, nombres en construcciones apelativas, muletillas, palabras conocidas internacionalmente como «no», «ok»; interjecciones como «ah», «wow», «oh», etc. (Georgakopoulou 2009: 27-28).

En las siguientes tablas puede verse que la Traducción1 y la Traducción2 se distinguen considerablemente entre sí:

\begin{tabular}{|c|c|c|c|}
\hline \multicolumn{4}{|c|}{$\begin{array}{l}\text { Hey, you know what we should all do? } \\
\text { We should play that game where everyone } \\
\text { says one thing they're thankful for. }\end{array}$} \\
\hline 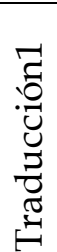 & $\begin{array}{l}\text { 00:00:38,180 --> 00:00:42,810 } \\
\text { Juguemos al juego en el que } \\
\text { cada uno agradece algo. }\end{array}$ & 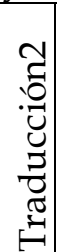 & $\begin{array}{l}\text { 00:00:36,897 --> 00:00:41,988 } \\
\text { Saben que deberíamos hacer? } \\
\text { Deberíamos } \\
\text { jugar ese juego donde todos dicen una } \\
\text { cosa por la cual estan agradecidos. }\end{array}$ \\
\hline
\end{tabular}

Como se puede apreciar, la Traducción1 es más corta pero suficiente para transmitir el mismo mensaje presente en la lengua original.

\begin{tabular}{|c|c|c|c|}
\hline \multicolumn{4}{|c|}{$\begin{array}{l}\text { So, Rachel, your mom tells me } \\
\text { you changed your major again? }\end{array}$} \\
\hline 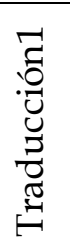 & $\begin{array}{l}\text { 00:13:02,490 --> 00:13:04,458 } \\
\text { ¿Cambiaste otra vez de carrera? }\end{array}$ & 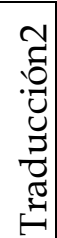 & $\begin{array}{l}\text { 00:13:01,731 --> 00:13:04,470 } \\
\text { Rachel, tu mama me dice que } \\
\text { cambiaste de nuevo de carrera. }\end{array}$ \\
\hline
\end{tabular}

En la Traducción1 no hay vocativo y se elude «tu mama me dice» ya que es irrelevante.

\begin{tabular}{|c|c|c|c|}
\hline \multicolumn{4}{|c|}{$\begin{array}{l}\text { Well, I don't care. } \\
\text { That turkey has to feed } 20 \text { people. } \\
\text { They're not going to eat it off your head. }\end{array}$} \\
\hline 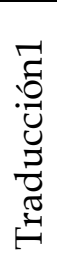 & $\begin{array}{l}\text { 00:05:56,481 --> 00:06:01,112 } \\
\text { Debe alimentar a } 20 \text { personas. } \\
\text { No lo comerán de tu cabeza. }\end{array}$ & 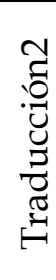 & $\begin{array}{l}\text { 00:05:56,075 --> 00:06:00,237 } \\
\text { Bueno, no me importa! ese pavo tienen } \\
\text { que } \\
\text { comerlo } 20 \text { personas en casa de mis } \\
\text { padres } \\
\text { y no se lo van a comer desde tu cabeza!. }\end{array}$ \\
\hline
\end{tabular}


En la Traducción1, la parte «Bueno, no me importa» se ha omitido porque se ve por la reacción del personaje que está enojada y le da todo igual. Por su parte, la Traducción2 no suprime nada del texto original, sino que hace un añadido respecto a la versión en inglés con «en casa de mis padres».

\section{Conclusión}

La mayoría de los científicos que investigan la subtitulación, como una de las ramas de la traducción audiovisual, están de acuerdo que el texto que aparece en la pantalla simultáneamente con la imagen, refleja, en la mayoría de los casos, los diálogos en la lengua meta que se presentan en la lengua de origen. El traductor debería respetar las normas técnicas para transmitir el mensaje adecuadamente. Las normas consisten en la restricción del texto traducido (un subtítulo) en un máximo de dos líneas de 40 caracteres que deberían estar en la pantalla seis segundos para que el espectador tenga el suficiente tiempo para leerlo y conectarlo con otros componentes del contenido audiovisual: la palabra dicha, la que se escucha, y la imagen. Esta sincronización es fundamental. También, existen unas reglas de ortografía específicas que se aplican en este tipo de traducción (las cursivas se usan para destacar las voces en off).

Teniendo en cuenta que el corpus de nuestro trabajo es la subtitulación amateur, hemos concluido que existen traducciones que respetan las reglas de la traducción audiovisual, pero, también, casos totalmente opuestos en los que la calidad de alguno de los aspectos de la traducción mencionados anteriormente, brillan por su ausencia. Comparando dos traducciones amateurs, hemos analizado la veracidad de las mismas centrándonos en varios puntos concretos, es decir, la traducción inexacta que provoca la transmisión de un mensaje equivocado; traducciones sin sentido; pérdida del humor por la mala traducción; pérdida de la naturalidad en el habla cotidiana y traducción del título. Por otro lado, como ya hemos mencionado, también hemos puesto el foco de atención en los aspectos formales de las traducciones, (aspectos técnicos, normas ortotipográficas y normas de estilo).

Las dos traducciones se diferencian muy claramente. Tras el estudio, podemos concluir que el traductor de la Traducción1 tiene cierta formación en subtitulación, pues conoce tanto las estrategias de traducción audiovisual como los aspectos formales de esta. Por el contrario, el traductor de la Traducción2 es amateur, en el sentido de la palabra enfatizado en este trabajo.

Adicionalmente, el problema que surge en la búsqueda de la traducción deseada es que no hay ninguna estrategia para encontrar una de buena calidad. Probablemente, la 
mayoría de ellas están hechas por parte de autores anónimos y su condición solo la podremos valorar cuando estemos viendo el contenido del material audiovisual o cuando hayamos terminado, es decir, cuando ya es tarde, en cierto modo. Precisamente, esto puede abrirnos nuevas e interesantes líneas de investigación, como indagar sobre cuáles pueden ser las estrategias para encontrar un subtítulo de buena calidad o tal vez analizar el contenido de las páginas web que ofrecen estos tipos de traducciones.

\section{BIBLIOGRAFÍA}

«Friends (1994)». Podnapisi.NET. Web. 10.02.2020.

«Friends». The Internet Movie Database. Web. 05.11.2019.

«Guía de episodios». Friends Peich. Web. 12.11.2019.

«The One with All the Thanksgivings». The Internet Movie Database. Web. 05.11.2019.

«The One with the Thanksgiving Flashbacks». Rotten Tomatos. Web. 05.11.2019.

Agost, Rosa. Traducción y doblaje: palabras, voces e imágenes. Barcelona: Ariel Practicum, 1999. Impreso.

Baker, Mona. Routledge Encyclopedia of Translation Studies. London: Routledge, 1998. Impreso.

Bogucki, Łukasz. «Amateur Subtitling on the Internet». Audiovisual Translation Language Transfer on Screen. Jorge Díaz Cintas y Gunilla Anderman (ed.). Nueva York: Palgrave Machmillan, 2009. 49-57. Impreso.

Carter, Bill. «'Friends' Finale's Audience Is the Fourth Biggest Ever». The New York Times, 8 May 2004. Web. 26.12.2019.

Castro Roig, Xosé. «El traductor de películas». La traducción para el doblaje y la subtitulación. Miguel Duro (coord.). Madrid: Cátedra, 2001. 267-298. Impreso.

Cuéllar Irala, Jana, y Andrea García-Falces. «Cultura y humor: traductores al borde de un ataque de nervios». Linguax. Revista de Lenguas Aplicadas 2 (2004): 3-31. Web. 10.11.2019.

Chaume Varela, Federico. Cine y traducción. Madrid: Cátedra, 2004. Impreso.

-. «Los estándares de calidad y la recepción de la traducción audiovisual». Puentes 6 (2005): 5-12. Impreso.

Díaz Cintas, Jorge. La traducción audiovisual: el subtitulado. Salamanca: Biblioteca de Traducción, 2001. Impreso.

- Teoría y práctica de la subtitulación: inglés/español. Barcelona: Ariel, 2003. Impreso 
Ferrer Simó, Mara Rosario. «Fansubs y scanlations: la influencia del aficionado en los criterios profesionales». Puentes: hacia nuevas investigaciones en la mediación intercultural 6 (2006): 27-43. Impreso.

García Luque, Francisca. «La compresión lingüística y la elisión en el subtitulado: estudios de "Le chateau de m amere"». Hermēneus. Revista de Traducción e Interpretación 7 (2005): 67-86. Impreso.

Georgakopoulou, Panayota. «Subtitling for the DVD Industry». Audiovisual Translation Language Transfer on Screen. Jorge Díaz Cintas y Gunilla Anderman (ed.). Nueva York: Palgrave Machmillan, 2009. 21-35. Impreso.

Gerding Salas, Constanza, et al. «Neología sintagmática anglicada en español: Calcos y préstamos». Revista signos 51.97 (2018): 175-192. Web. 19.10.2019.

Knapp, Marc. La comunicación no verbal: el cuerpo y el entorno. Traducida por Marco Aurelio Galmarini. Barcelona: Paidós, 2009. Impreso.

Littré, Émile. «amateur». Dictionnaire de la langue française. Littre.org. Web. 22.02.2020.

Matte Bon, Francisco. Gramática comunicativa del español. Tomo 1. Madrid: Edelsa, 2009. Impreso.

Zabalbeascoa, Patrick. «La traducción del humor en textos audiovisuales». La traducción para el doblaje y la subtitulación. Miguel Duro (coord.). Madrid: Cátedra, 2001. 251263. Impreso. 